\title{
What can DTI add in acute ischemic stroke patients?
}

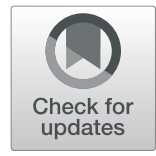

Bahaa Eldin Mahmoud ${ }^{1}$, Mohammad Edrees Mohammad² and Dalia K. Serour ${ }^{\text {** }}$

\begin{abstract}
Background: Cerebrovascular stroke is one of the leading causes of death worldwide. Imaging with conventional MR techniques cannot provide reliable information as regard the integrity of the white matter tracts and therefore limiting its ability to predict the clinical outcome. While prediction of the motor outcome becomes more crucial for determining the specific rehabilitation strategies and final clinical outcomes, the purpose of this study was to assess the value of diffusion tensor MR imaging in patients with acute ischemic stroke as a prognostic imaging modality to predict the clinical outcome.

Results: A significant statistical association was found between the tractography findings and the clinical score at admission ( $p$ 0.0005) and the clinical recovery after 3 months ( $p 0.001$ ). Residual neurological deficits were found in patients with disrupted tracts; on the other hand, near complete clinical recovery was found in patients with non-disrupted tracts. Also, significant statistical association was found between the degree of FA reduction in the affected tracts and the clinical score at admission ( $p$ 0.001) and the clinical recovery after 3 months ( $p 0.01$ ). Correlation between the FA values at the site of infarctions and the corresponding area of the brain on the contralateral side revealed significant statistical difference.

Conclusion: DTI offers a potential tool for prediction of the clinical outcome of acute stroke patients as it can detect the microstructural changes in the white matter tracts affected by the ischemic lesions which cannot be detected by conventional MRI and therefore can help in determining the rehabilitation strategy
\end{abstract}

Keywords: Diffusion tensor imaging, Tractography, Acute stroke, Prognosis, White matter tracts

\section{Background}

Acute cerebrovascular stroke is one of the leading causes of mortality and disability worldwide especially in elderly population [1]. Stroke is a broad clinical tem used to describe acute ischemic arterial infarcts, venous infarcts, and intracranial hemorrhage [2]. The mechanism of brain damage includes neuronal cell death and tissue loss caused by hypoperfusion and the hypoxic brain damage resulting in collapse of osmotic regulation and cytotoxic edema [3]. The brain stem affection in posterior circulation stroke has been associated with high morbidity and mortality, with the mortality often reaching up to $30-70 \%$ [4].

Currently, the most widely used powerful diagnostic tool for acute stroke is the diffusion MRI (DWI) [1]. Diffusion images can be rapidly acquired and interpreted

\footnotetext{
* Correspondence: daliakhaled80@yahoo.com

${ }^{1}$ Department of Diagnostic and Interventional Radiology, Cairo University, Giza, Egypt

Full list of author information is available at the end of the article
}

over a few minutes and can guide the reperfusion therapies early after onset of symptoms [5].

DWI is an MRI technique sensitized to the Brownian motion of the water molecules in human tissues. As the white matter tracts of the brain are presented in different directions, the diffusion in such tracts is not uniform but considered as anisotropic, along the direction of the various fiber tracts [2]. Diffusion tensor imaging (DTI) is a recent non-invasive functional MR technique used to study the microstructural changes of the brain tissue. The FA (fractional anisotropy) detects the directionality of diffusion of water molecules inside the tissue. Abnormalities in the white matter causing loss of axonal organization produce lower FA values [6].

More than $50 \%$ of acute stroke patients experiencing residual neurological deficits with motor weakness of the upper limbs considered as the most frequent complication of stroke [7]. The clinical severity of acute stroke early in disease seems to have an influence on the overall stroke outcome. Microstructural damage to the white 
matter tracts and loss of the structural integrity affect the proper flow of neuronal information across the tracts, and the integrity of the corticospinal tract (CST) is critical for motor recovery [4]. Therefore, early evaluation of the integrity of the white matter tracts in acute stroke patients would assist in prediction of the clinical outcome, determining the optimal treatment and the appropriate rehabilitation strategies [7]. The current conventional MRI sequences alone cannot provide sufficient reliable information about the integrity of the white matter tracts and thereby limiting their ability to predict the clinical outcome [8].

The purpose of our study is to evaluate the role of DTI and fiber tractography in the prediction of clinical recovery after acute cerebrovascular stroke.

\section{Methods}

A prospective study included 66 patients presented with acute ischemic stroke in the period between October 2017 and November 2018. Six patients were excluded due to motion artifact that degraded the quality of the DTI maps and the final cohort was 60 patients. Written informed consents were obtained from all patients. The study is IRB approved.

MRI with diffusion tensor imaging was done for all patients within the first 2 days after the clinical presentation. The clinical neurological deficits were assessed by a neurology specialist with a 10-year experience using the National Institutes of Health Stroke Scale (NIHSS) on patient's admission. The NIHSS is a systematic assessment tool providing a quantitative measure of stroke-related neurologic deficit. The NIHSS is widely used to evaluate the acuity of stroke patients, determine appropriate treatment, and predict the patient outcome (Fig. 1) [9].

The patients were then clinically followed up after 3 months to detect any residual neurological deficits. Neurological improvement was defined as a decrease in the NIHSS score after 3 months compared to that on admission.

Exclusion criteria:

- Patients with intracerebral hemorrhage.

- Patients with absolute MRI contraindications.

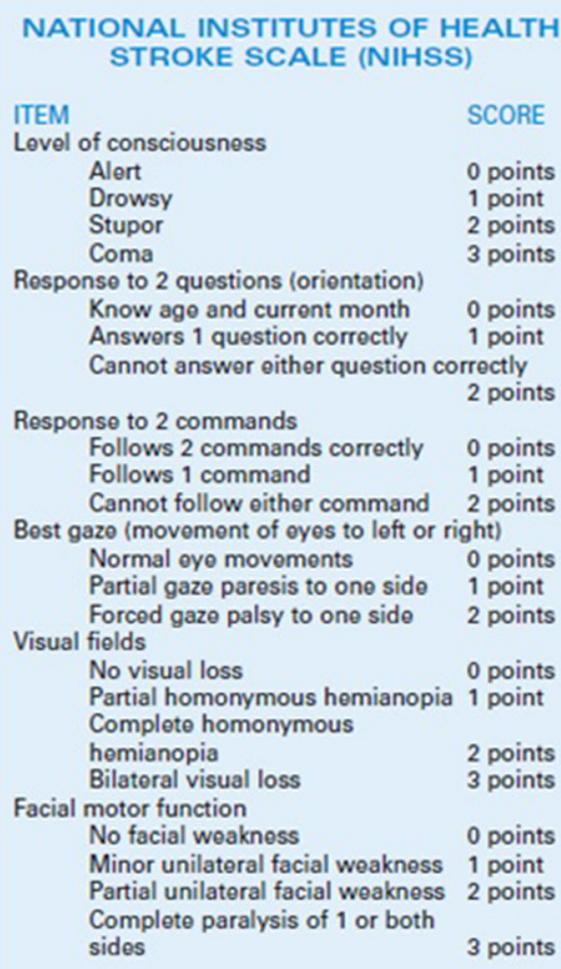

Upper-extremity motor function (right and left scored independently $0-8$ points) Normal movement 0 points Drift of upper extremity 1 point Some offort against gravity 2 points No effort against gravity but moves 3 points No movement 4 points Lower-extremity motor function (right and loft scored independently $0-8$ points) Normal movement $\quad 0$ points Drift of lower extremity 1 point Some effort against gravity 2 points No effort against gravity but moves 3 points No movement 4 points

Limb ataxia (cannot be tested in presence of paresis) Ataxia present in 1 limb Ataxia prosent in 2 limbs 2 points Sensory function No sensory loss $\quad 0$ points Mild-to-moderate sensory loss 1 point Severe-to-total sensory loss 2 points 
Table 1 Correlation between the degree of FA reduction and NIHSS on admission

\begin{tabular}{llll}
\hline NIHSS & FA & & \\
\cline { 2 - 4 } & Mild $(n=5)$ & Moderate $(n=36)$ & Severe $(n=19)$ \\
\hline Mild $(n=10)$ & 0 & 2 & 8 \\
Moderate $(n=40)$ & 5 & 24 & 11 \\
Severe $(n=10)$ & 0 & 10 & 0 \\
& & & $\mathrm{P} 0.001$ \\
\hline
\end{tabular}

MRI technique was done using a standard 1.5 Tesla unit (Achiva, Philips) using a standard head coil. The obtained sequences were axial T1W, T2W, FLAIR, diffusionweighted imaging, and diffusion tensor. The diffusion tensor imaging consisted of a single shot, spin-echo echoplanar sequence in 12 encoding directions, and a diffusion weighting factor of $800 \mathrm{~s} / \mathrm{mm}^{2}$, TR $8000 \mathrm{~ms}$, TE $67 \mathrm{~ms}$, flip angle 90, matrix $112 \times 110$, FOV $210 \times 236 \mathrm{~mm}$, and number of excitations, 2 and slice thickness, 2.0/00.

Post processing was done on the MR workstation (Phillips Extended MR Workspace, 2.6.3.5 Netherlands).

The maps obtained were as follows:

1. Grey scale FA maps.

2. Directionally encoded color FA maps.

3. 3D fiber tractography was performed using multiROI technique based on known anatomy. Regions of interest (ROIs) were drawn in the unaffected portion of the white matter tracts, and the software detects the white matter tracts that passed through the ROIs.

Freehand drawings of the regions of interest (ROIs) were made at the FA color map overlaid on T2 or FLAIR images. Measurements were performed at the site of infarction and the corresponding area at the contralateral hemisphere.

For classification of the patterns of white matter tract affection as detected by 3D fiber tractography, we followed the classification of Witwer et al. [10]. The tracts were classified into preserved, displaced, or disrupted.

Disrupted tract: if the tract showed marked reduction of the anisotropy so that it could not be identified on

Table 2 Correlation between the tractography findings and NIHSS on admission

\begin{tabular}{lll}
\hline NIHSS & Tractography \\
\cline { 2 - 3 } & Disrupted $(n=38)$ & Non-disrupted $(n=22)$ \\
\hline Mild $(n=10)$ & 1 & 9 \\
Moderate $(n=40)$ & 29 & 11 \\
Severe $(n=10)$ & 8 & 2 \\
& & $P 0.0005$ \\
\hline
\end{tabular}

Table 3 Correlation between the degree of FA reduction and NIHSS after 3 months

\begin{tabular}{llll}
\hline NIHSS & FA & & \\
\cline { 2 - 4 } & Mild $(n=5)$ & Moderate $(n=36)$ & Severe $(n=19)$ \\
\hline Mild $(n=26)$ & 5 & 15 & 6 \\
Moderate $(n=34)$ & 0 & 21 & 13 \\
Severe $(n=0)$ & 0 & 0 & 0 \\
& & & $P 0.01$ \\
\hline
\end{tabular}

the FA maps and could not be traced by fiber tracking algorithm.

Displaced tract: if the tract showed abnormal location or abnormal orientation, but it maintains its normal anisotropy when compared to the corresponding contralateral tract.

Preserved tract: if the tract maintains its normal location, orientation, and anisotropy compared to the corresponding contralateral normal tract.

In cases with more than one tract affection, it is considered disrupted if at least one tract is disrupted.

The patients were classified according to the following:

$\bigcirc$ The clinical score (NIHSS): Mild (1-4), moderate (5-10), and severe (> 10).

$\bigcirc$ The degree of FA reduction of the white matter tracts at the site of infarction: mild (0.4), moderate $(0.2-0.3)$, and severe $(0.1)$.

$\mathrm{T}$ The pattern of WM tract affection whether disrupted or non-disrupted (either preserved or displaced). Disrupted (if at least one tract is disrupted).

\section{Statistical analysis}

$\bigcirc$ Results are expressed as mean \pm standard deviation or number (\%).

$\bigcirc$ Association between different parameters was performed using chi-square test.

\section{Results}

Sixty six patients were included in the study (38 males and 28 females). Six patients were excluded due to motion artifact that degraded the quality of the DTI maps and the final cohort was 60 patients

Table 4 Correlation between the tractography findings and NIHSS after 3 months

\begin{tabular}{lll}
\hline NIHSS & Tractography & \\
\cline { 2 - 3 } & Disrupted $(n=38)$ & Non-disrupted $(n=22)$ \\
\hline Mild $(n=26)$ & 8 & 18 \\
Moderate $(n=34)$ & 30 & 4 \\
Severe $(n=0)$ & 0 & 0 \\
& & $P 0.001$ \\
\hline
\end{tabular}


(34 males and 26 females). The patients' ages ranged between 24 and 75 years, and the mean age was 58.2 years.

The correlation between the degree of FA reduction and the NIHSS on admission is summarized in Table 1. The five patients in the mild FA group were all belonging to the moderate NIHSS group. While among the 36 patients in the moderate FA group, two patients (5.5\%) fall in the mild NIHSS group, 24 patients (66.7\%) in the moderate group, and 10 patients $(27.8 \%)$ in the severe group. In the severe FA group, eight patients (42.2\%) were in the mild NIHSS group and 11 patients (57.8\%) were in the moderate group. The results were statistically significant $(p=0.001)$ denoting that, the more the degree of FA reduction, the more the brain damage and the higher the clinical severity.
The correlation between the pattern of white matter tract affection as detected by 3D fiber tractography and the NIHSS on admission is summarized in Table 2 . Among the 38 patients with disrupted white matter tracts, 1 patient (2.6\%) fall in the mild NIHSS group, 29 patients $(76.3 \%)$ in the moderate group, and 8 patients (21.1\%) in the severe group. Whereas from the $22 \mathrm{pa}-$ tients in the non-disrupted group, 9 patients $(40.9 \%)$ were in the mild NIHSS group and 11 patients $(50 \%)$ were in the moderate group and 2 in the severe group (9.1\%). The results were highly statistically significant $(p=0.0005)$.

The correlation between the degree of FA reduction and the NIHSS after 3 months is summarized in Table 3. All the 5 patients in the mild FA group were belonging to the mild NIHSS group. While among the 36 patients in the

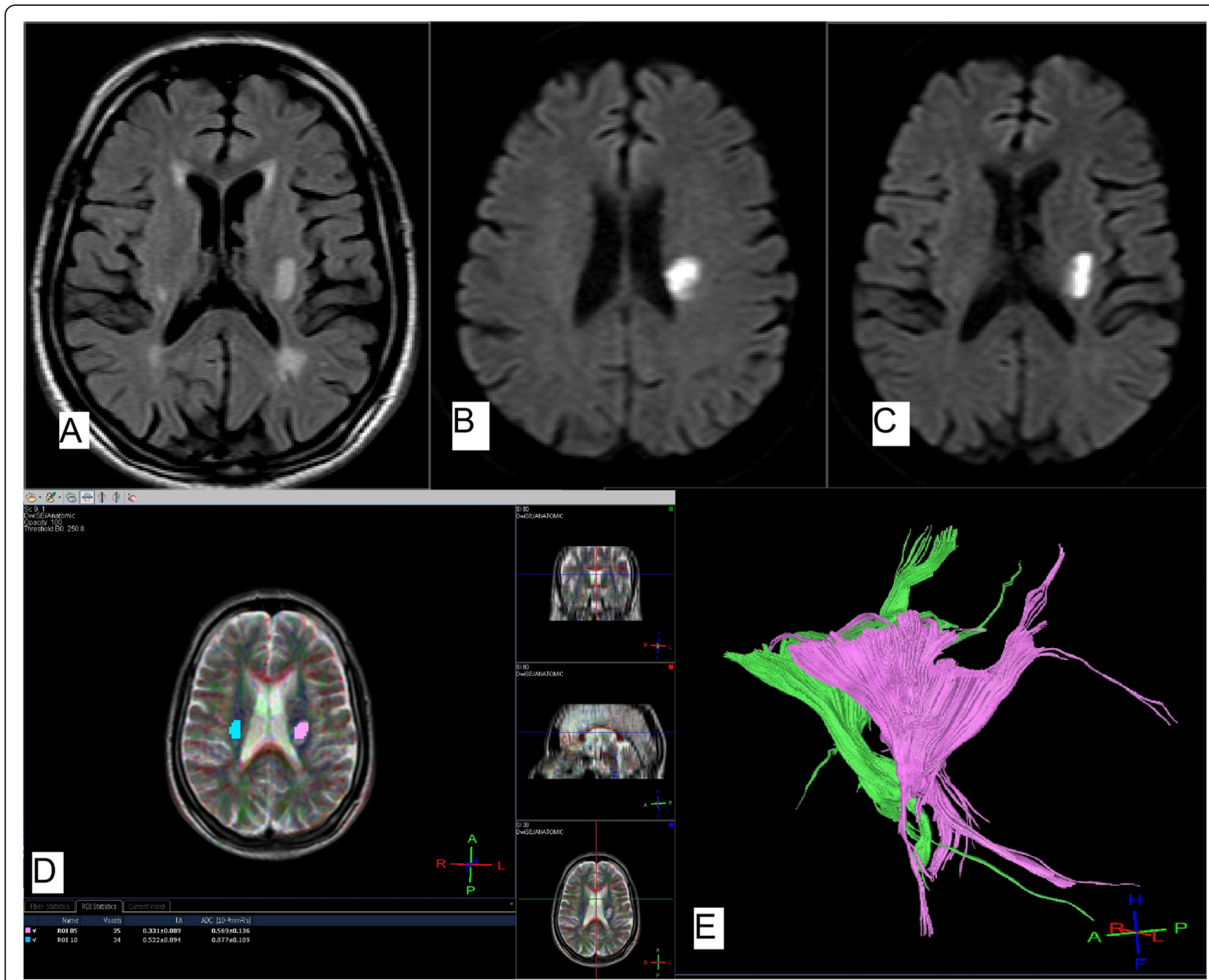

Fig. 2 A 75-year-old male patient, diabetic and hypertensive, presented with right hemiplegia. NIHSS on admission was 9. a Axial FLAIR and b, c axial diffusion WIs (b1000) show left corona radiata and posterior limb of internal capsule area of high FLAIR signal and restricted diffusion in keeping with acute infarct. $\mathbf{d}$ Axial color-coded FA map overlaid on axial T2 images showing reduced FA value at the site of infarct (0.3) compared to the contralateral normal side (0.5). e 3D fiber tractography of the CST showing disrupted some fibers of the left CST. The patient shows minimal clinical improvement with residual motor weakness. NIHSS after 3 months was 8 
moderate FA group, 15 patients (41.6\%) fall in the mild NIHSS group and 21 patients $(58.4 \%)$ in the moderate group. Whereas in the severe FA group, 6 patients (31.6\%) were in the mild NIHSS group and 13 (68.4\%) were in the moderate group. The results were statistically significant $(p=0.01)$ denoting that the more the degree of FA reduction and brain damage, the more the residual neurological deficits observed in the patients.

The correlation between the pattern of white matter tract affection as detected by 3D fiber tractography and the NIHSS in the clinical follow-up after 3 months is summarized in Table 4. Among the 38 patients with disrupted white matter tracts, 8 patients $(21.1 \%)$ fall in the mild NIHSS group and 30 patients $(78.9 \%)$ in the moderate group. Whereas from the 22 patients in the nondisrupted group, 18 patients $(81.8 \%)$ were in the mild NIHSS group and 4 patients (18.2\%) were in the moderate group. The results were highly statistically significant $(p=0.001)$ denoting that the residual neurological deficits were higher in the patients with disrupted white matter tracts (Figs. 2 and 3) than those with displaced and preserved tracts (Figs. 4 and 5).

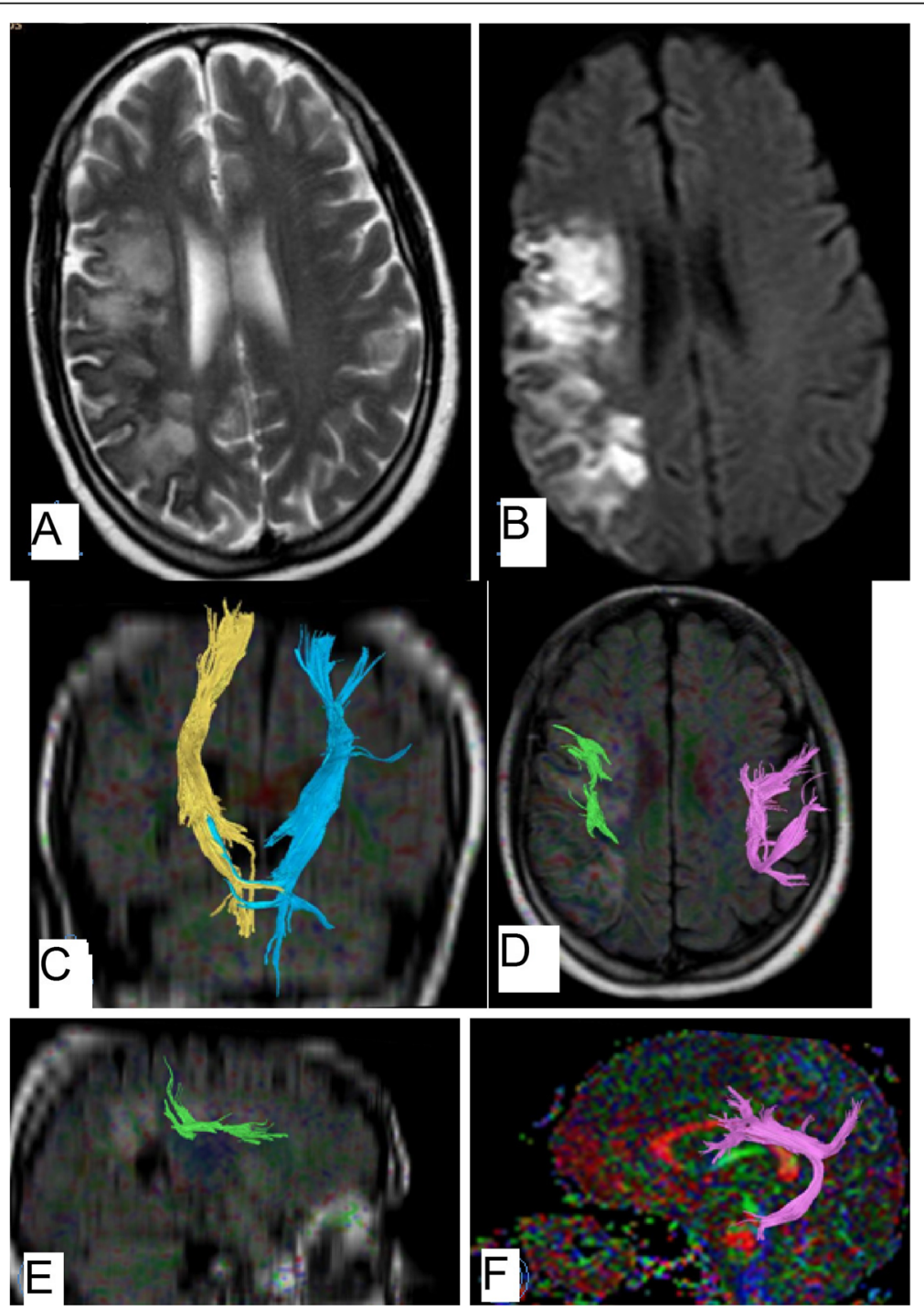

Fig. 3 A 57-year-old female patient, diabetic and hypertensive, presented with dysarthria. NIHSS on admission was 7 a axial T2 b axial diffusion WIs (b1000) show right fronto-parietal area of high signal and restricted diffusion in keeping with acute infarct. c Coronal FA color-coded map overlaid over coronal FLAIR image with fiber tractography of the CST that appear preserved. $\mathbf{d}$ Axial FA color-coded map overlaid over axial FLAIR image with tractography of the acurate fasciculus. e Sagittal FA color-coded map overlaid over sagittal FLAIR image with tractography of the right arcuate fasciculus. f Sagittal FA color-coded map with tractography of the left arcuate fasciculus. Note the disrupted right arcuate fasciculus (green) compared to the left one (pink). FA value at the site of infarct (0.3) compared to the contralateral normal side (0.4). The patient shows mild clinical improvement with residual speech difficulties. NIHSS after 3 months was 5 
By comparing the mean FA at the site of infarction and the mean FA of the corresponding area on the contralateral hemisphere, we found a significant statistical difference ( $p$ 0.001) with evidence of significant FA reduction at the site of infarct compared to the normal contralateral side indicating brain damage.

The previous results indicating that DTI with FA measurements and 3D fiber tractography can predict the clinical outcome of acute stroke patients (the more the severity of the clinical presentation, the more the damage of the white matter tracts).

\section{Discussion}

Acute cerebrovascular stroke is one of the leading causes of mortality and disability worldwide especially in elderly population [1]. One of the commonest neurological deficits after acute stroke is upper-limb motor impairment, which can have a significant impact on disability and health [11]. The role of imaging with conventional MRI and DWI is limited to the early diagnosis of the infracted tissue, while diffusion tensor imaging has shown promising results in the evaluation of pathological microstructural changes in ischemic tissues and prediction of the clinical outcome $[12,13]$.

The purpose of this study was to assess the value of diffusion tensor MR imaging in patients with acute ischemic stroke as a prognostic imaging modality to predict the clinical outcome.

A significant statistical correlation was found between the degree of FA reduction and the NIHSS score on admission and that on follow-up after 3 months. This indicates that the more the degree of FA reduction, the higher the clinical severity and the more the residual neurological deficits observed in the patients, thus reflecting more brain damage. We also found a significant statistical difference between the FA reduction at the site of infarction compared to the contralateral hemisphere. Our results agreed with Abdeldayem et al. [14] who found significant correlation between the FA with the patient clinical outcome and recovery after 3 months assessed by the Modified Rankin Score (MRS). This was also matched with the results of Song et al. [15] who found that the higher FA values were correlated with better neurological recovery and comparing the ipsilateral and contralateral posterior limb of the internal capsule can significantly predict the motor outcome. Also, in another study by Kusano et al. [16] done on 18 patients with cerebrovascular stroke, it was found that the FA at the side of infarction was significantly lower than the unaffected side and significantly correlated with the MRS score.

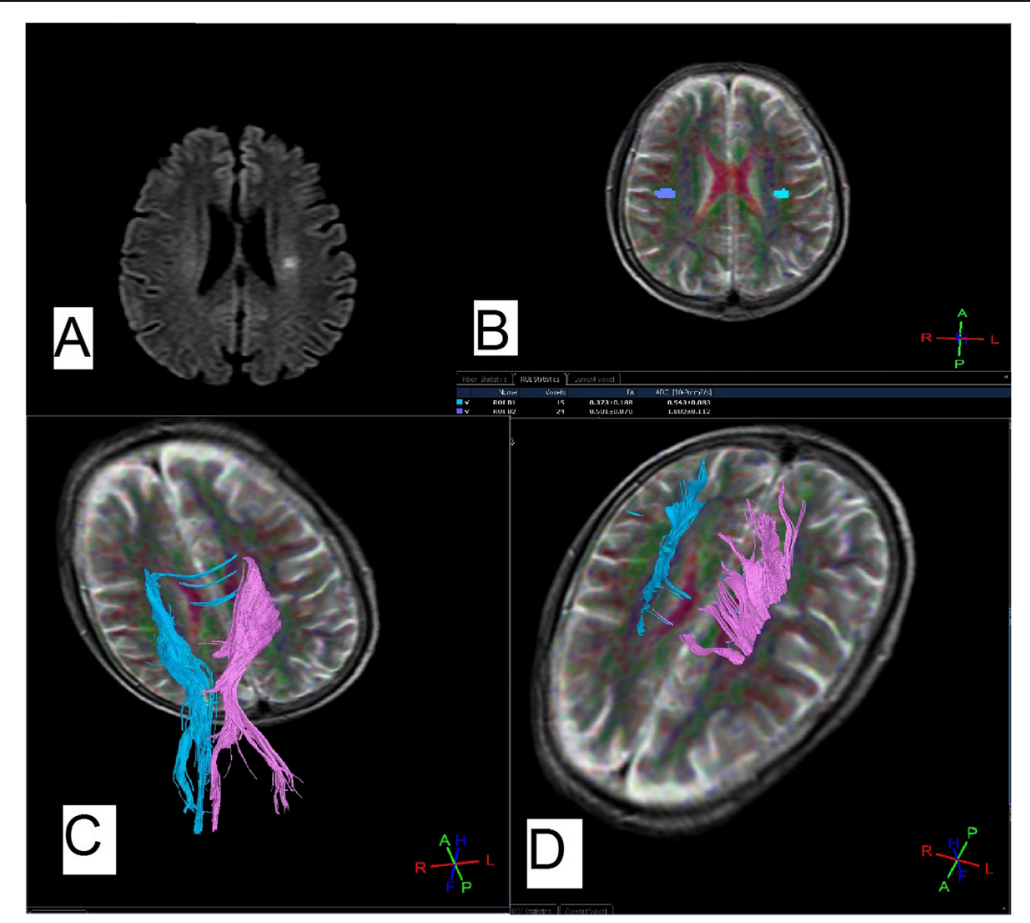

Fig. 4 A 64-year-old male patient, diabetic and hypertensive, presented with mild right-sided weakness. NIHSS on admission was 5 a axial diffusion Wls (b1000) show small left parietal acute infarct. $\mathbf{b}$ Axial color-coded FA map overlaid on axial T2 images showing reduced FA value at the site of infarct (0.3) compared to the contralateral normal side (0.5) c $\mathbf{d}$ axial FA color-coded map overlaid over axial T2 image with 3D fiber tractography of the CST that appear preserved. The patient shows a significant clinical improvement with residual speech difficulties. NIHSS after 3 months was 2 

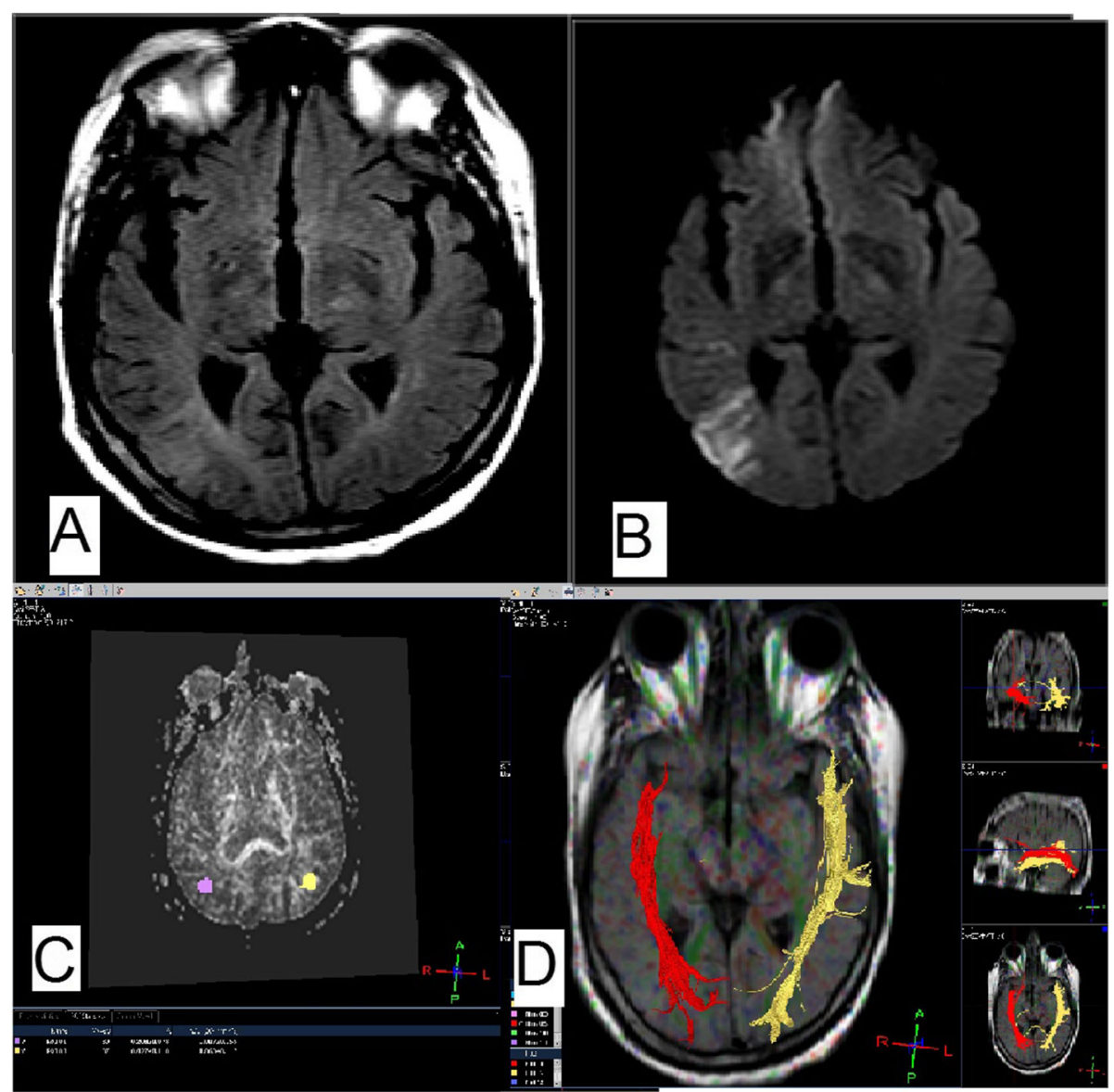

Fig. 5 A 50-year-old male patient, diabetic and hypertensive, presented with DCL. NIHSS on admission was 10 a axial FLAIR. b Axial diffusion WIs (b1000) show right occipital acute infarct. c Grey scale FA map showing reduced FA value at the site of infarct (0.2) compared to the contralateral normal side (0.3) $\mathbf{d}$ axial FA color-coded map overlaid over axial FLAIR image with 3D fiber tractography of the inferior longitudinal fasciculus that appear preserved. The patient shows significant clinical improvement with residual speech difficulties. NIHSS after 3 months was 4

However, our results were in controversy with Elkholy et al. [2] who found insignificant statistical correlation between the degree of FA reduction and the NIHSS score. This could be explained by the different methodology between the two studies, as they included some cases with chronic infarcts.

In our study, we also found a significant statistical correlation between the pattern of white matter tract affection as detected by 3D fiber tractography and the NIHSS score on admission and that on follow-up after 3 months. This indicates that the clinical severity on admission and the residual neurological deficits were higher in the patients with disrupted white matter tracts than those with displaced and preserved tracts. Our results agreed with Elkholy et al. [2] who studies 50 patients with stroke and found that $100 \%$ of the patients with disrupted tracts had residual neurological deficits, whereas the patients with non-disrupted tracts (displaced and preserved) had near complete clinical recovery and Abdeldayem et al. [14] who found that all the patients in their study presented with non-disrupted tracts showed good clinical outcome while $86.4 \%$ of the patients with disrupted fibers showed poor outcome.

Our results were also agreed with Kwon et al. [17] who found a direct relation between the degree of motor disability and the tractography findings with $p$ value $<0.05$. Also with Shereen et al. [18] who found a significant statistical correlation between the findings of fibers tractography and the motor changes as well as the clinical outcome.

In another study done by Parmar et al. [8] on 11 patients with stroke, they found that all the patients with disrupted tracts had residual neurological deficits on the clinical follow-up while patients with non-disrupted (displaced) tracts had near complete neurological recovery.

Although the study has several strengths, it also has some weak points and limitations that prompt further investigations. The sample size is relatively small. Another limitation is that the follow-up was done only using the NIHSS score while MRI was not performed again to detect any improvement or changes in the DTI 
measures. However, this was related to the clinical practice as many patients may refuse to repeat the MRI, so we depends on the clinical follow-up only as done in previous studies. The clinical follow-up was done using the NIHSS score, unlike other studies that used another clinical assessment method which is the MRS. One of the limitations is the variations in the site of the lesions and the affected tracts, and thus, the variable clinical presentation depending on the affected tracts; however, our main aim was to evaluate the microstructural white matter changes regardless which tract is affected.

\section{Conclusion}

Diffusion tensor imaging with fiber tractography is a powerful functional MRI technique that can detect the microstructural changes and integrity of the white matter tracts in patients with acute cerebrovascular stroke; thus, it can predict the clinical outcome and can guide the rehabilitation strategy.

\section{Abbreviations}

CST: Corticospinal tract; DTI: Diffusion tensor imaging; DWl: Diffusionweighted images; FA: Fractional anisotropy; MRS: Modified Rankin Score; NIHSS: National Institutes of Health Stroke Scale; ROI: Region of interest; WM: White matter

\section{Acknowledgements}

Not applicable.

\section{Authors' contributions}

BEM was the editor of the manuscript, participated in the study design, and performed the DTI analysis and FA measurements. MEM helped in patient collection and clinical assessment. DKS put the idea of the study; participated in the study design, FA measurements, and DTI analysis; and performed the statistical analysis. All authors read and approved the final manuscript.

\section{Funding}

Not applicable (no funding received for this study).

\section{Availability of data and materials}

All the datasets used and analyzed in this study are available with the corresponding author on reasonable request.

\section{Ethics approval and consent to participate}

Written informed consent was signed by all patients before the examination. The study was approved by the research committee of the Faculty of Medicine, Cairo University.

\section{Consent for publication}

All patients included in this research were fully conscious and older than 16 years old and gave written informed consent.

\section{Competing interests}

The authors declare that they have no competing interests.

\section{Author details}

${ }^{1}$ Department of Diagnostic and Interventional Radiology, Cairo University, Giza, Egypt. ²Department of Neurology, Cairo University, Giza, Egypt.
Received: 9 August 2019 Accepted: 24 September 2019

Published online: 02 December 2019

\section{References}

1. Moura LM, Luccas R, Paiva JPQ et al (2019) Diffusion tensor imaging biomarkers to predict motor outcomes in stroke: a narrative review. Front Neurol 10:445. https://doi.org/10.3389/fneur.2019.00445

2. Elkholy SF, Khalil ME, Elbasmi AA et al (2015) Diffusion tensor magnetic resonance imaging in assessment of prognostic outcome of stroke patients. EJRNM 46(3):707-713

3. Grotta J, Albers G, Broderick JP, et al. (2016) Stroke: pathophysiology, diagnosis, and management. Elsevier Health Sciences.

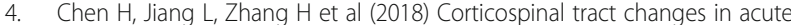
brainstem ischemic stroke patients: a diffusion kurtosis imaging study. Neurol India 66(3):726-732. https://doi.org/10.4103/0028-3886.232281

5. Nogueira RG, Jadhav AP, Haussen DC et al (2018) Thrombectomy 6 to 24 hours after stroke with a mismatch between deficit and infarct. N Engl J Med 378:11-21. https://doi.org/10.1056/NEJMoa1706442

6. Razek AAKA, El-Serougy L, Abdelsalam M et al (2018) Differentiation of residual/recurrent gliomas from postradiation necrosis with arterial spin labeling and diffusion tensor magnetic resonance imaging-derived metrics. Neuroradiology 60:169-177

7. Kumar P, Kathuria P, Nair P, Prasad K (2016) Prediction of upper limb motor recovery after subacute ischemic stroke using diffusion tensor imaging: a systematic review and meta-analysis. Journal of Stroke 18(1):50-59 https:// doi.org/10.5853/jos.2015.01186

8. Parmar H, Golay X, Lee KE et al (2006) Early experiences with diffusion tensor imaging and magnetic resonance tractography in stroke patients. Singap Med J 47(3):198-203

9. Ortiz, Gustavo \& L. Sacco, Ralph. (2008). National Institutes of Health Stroke Scale (NIHSS). https://doi.org/10.1002/9780471462422.eoct400.

10. Witwer BP, Moftakhar R, Hasan KM et al (2002) Diffusion-tensor imaging of white matter tracts in patients with cerebral neoplasm. J Neurosurg 97:568-575

11. Coupar F, Pollock A, Rowe P et al (2012) Predictors of upper limb recovery after stroke: a systematic review and meta-analysis. Clin Rehabil 26:291-313. https://doi.org/10.1177/0269215511420305

12. Zhang $X$, Yan $Y$, Tong $F$ et al (2018) Progressive assessment of ischemic injury to white matter using diffusion tensor imaging: a preliminary study of a macaque model of stroke. The Open Neuroimaging Journal 12:30-41

13. Pitkonen M, Abo-Ramadan U, Marinkovic I et al (2012) Long-term evolution of diffusion tensor indices after temporary experimental ischemic stroke in rats. Brain Res 1445:103-110

14. Abdeldayem EH, Osman AM, Tamer MR (2017) Relative fractional anisotropy value using DTI: can it help as a prognostic tool in anticipating the recovery of stroke patients? The Egyptian Journal of Radiology and Nuclear Medicine 48:1019-1025

15. Song J, Nair VA, Young BM et al (2015) DTI measures track and predict motor function outcomes in stroke rehabilitation utilizing $\mathrm{BCl}$ technology. Front Hum Neurosci 9:195

16. Kusano $Y$, Seguchi T, Horiuchi T (2009) Prediction of functional outcome in acute cerebral hemorrhage using diffusion tensor imaging at 3T: a prospective study. AJNR 30(8):1561-1565

17. Kwon YH, Jeoung YJ, Lee J et al (2012) Predictability of motor outcome according to the time of diffusion tensor imaging in patients with cerebral infarct. Neuroradiology 54(7):691-697

18. Shereen A, Nemkul N, Yang D et al (2011) Ex vivo diffusion tensor imaging and neuropath logical correlation in a murine model of hypoxia-ischemiainduced thrombotic stroke. J Cereb Blood Flow Metab 31:1155-1169

\section{Publisher's Note}

Springer Nature remains neutral with regard to jurisdictional claims in published maps and institutional affiliations. 